\title{
On the Fading Channel Performance of Temporal-Spatial Filters for CDMA
}

\author{
Aylin Yener \\ EECS, Lehigh University \\ yener@eecs.lehigh.edu
}

\author{
Sennur Ulukus \\ AT\&T Labs-Research \\ ulukus@research.att.com
}

\begin{abstract}
CDMA system performance can be enhanced by interference management techniques. We consider the uplink of a CDMA system that employs two such techniques: temporal multiuser detection and spatial antenna array heamforming. Recently, temporal-spatial MMSE type receivers with several different criteria have been proposed for a static channel. In this work, we consider such MMSE filters assuming a multiple time-scale fading channel model. It is shown that under the given channel conditions and system assumptions, three recently proposed MMSE type receivers; the unconstrained optimum MMSE detector, the constrained optimum MMSE detector and the cascaded temporal-spatial MMSE detector, are identical and the resulting combined receiver employs an MMSE multiuser detector at the output of each array element (temporal MMSE filter) followed by an MRC (spatial matched filter).
\end{abstract}

\section{Introduction}

It is well known that CDMA systems are interference limited and interference management is necessary to enhance the system capacity. To this end, a number of receiver and/or transmitter based interference management techniques are considered for CDMA systems; see for example $[7,10,12-14,18]$. In this work, we concentrate on the two commonly used interference management methods: multiuser detection and receiver beamforming. Both methods aim at suppressing or cancelling the interference using receiver signal processing; multiuser detection exploits the temporal structure whereas beamforming exploits the spatial structure of the interference for interference management $[6,14]$.

Multiuser detection [14] performs temporal filtering of the received signal to effectively suppress the multiple access interference. The optimum multiuser detector has been shown to be exponentially complex in the number of users, and a number of low complexity suboptimum receivers have been proposed following this development $[4,5]$. Increas- ing the capacity of CDMA systems by employing antenna arrays at the base station has been proposed in [7], where the outputs of the multiple antenna array elements are combined to make bit decisions for the user. In [7], matched filter receivers are assumed in the temporal domain for each user and the array observations are combined via a filter that is matched to the array response of the user, i.e., single user processing is employed in both domains.

Another method of capacity enhancement which utilizes the spatial diversity is space-time processing for CDMA which traditionally refers to receiver beamforming (space processing) and multipath combining (time processing) [8]. The received signals from different paths and antennas are combined to better decode the desired user's bits. However, the inherent structure of the multiple access interference is not exploited, i.e., no multiuser detection is employed $[1,3,16]$. Reference [15] addresses the derivation of the sufficient statistics and the optimum and some linear suboptimum multiuser detectors when an antenna array is present at the receiver for a multipath channel.

Recently, possible receiver structures were studied for the case where both multiuser detection and beamforming are employed to further increase the uplink capacity of a CDMA system $[17,18]$. Assuming linear processing in both the temporal and the spatial domains, the temporal-spatial filters were denoted by two-dimensional matrix filters and several possible filter structures, all based on minimum mean squared error (MMSE) criterion, were developed. In particular, the unconstrained jointly optimal temporalspatial filter, and a less complex constrained optimum filter, which was developed by constraining the joint receiver filter matrix to be of rank 1, were considered [18]. Both filters minimize the mean squared error (MSE) between the information bit and the filter output of a desired user. References $[17,18]$ assumed a static channel and that all users' channel gains to all antennas are tracked at the receiver. In this paper, we will concentrate on the case where users experience multiple time-scale fading. As in [19], we differentiate the large-scale fading coefficients which are slowly changing and can be estimated, from the small-scale fading coefficients to all array elements which may be difficult for the re- 
ceiver to track. Given the available statistics at the receiver for this system model, we derive the jointly optimal temporal and spatial filter that minimizes the mean squared error (MSE) between the information bit and the filter output of a desired user. We show that this filter has rank 1 and therefore coincides with the constrained optimum (rank-1) filter. We also consider the cascaded temporal-spatial MMSE filter proposed in [17], under these channel conditions. We show that the cascaded temporal-spatial filter which is obtained by solving two MMSE problems in tandem in the temporal and spatial order, also coincides with the two aforementioned filters; i.e., minimization of the end-to-end MSE is equivalent to minimizing the MSE in the temporal domain followed by in the spatial domain. Furthermore, the resulting separable temporal-spatial filter is of the form of a temporal MMSE filter followed by a maximum-ratio combiner (MRC).

\section{System Model}

We consider a $K$-user, single cell DS-CDMA system where each user is assigned a unique signature sequence. For clarity of exposition, we assume a synchronous system with processing gain $N$. At the base station, an antenna array of $L$ elements is employed. The received signal at the output of the antenna array at the base station is:

$$
\mathbf{r}(t)=\sum_{j=1}^{K} \sqrt{p_{j}} \sqrt{h_{j}} b_{j} s_{j}(t) \mathbf{a}_{j}+\mathbf{n}(t)
$$

where $p_{j}, b_{j}, s_{j}(t)$ are the power, the bit, and the (temporal) signature of user $\mathrm{j}$. The large-scale (long term) fading coefficient for user $\mathrm{j}$ is denoted by $h_{j}$ whereas the small-scale (short-term) fading coefficient of user $j$ to the $l$ th antenna array element is denoted by $a_{j l}$ giving rise to $\mathbf{a}_{j}$, the array response vector (spatial signature) of user $j$. We assume that the temporal signatures of the users have unit energy and are of the following form

$$
s_{j}(t)=\sum_{l=1}^{N} s_{j}^{(l)} \psi\left(t-(l-1) T_{c}\right)
$$

where $\psi(t)$ is the chip waveform, $T_{c}$ is the chip duration. Chip matched filtering and sampling the received signal $\mathbf{r}(t)$ at the $k$ th chip interval, we obtain an $L$-dimensional observation vector $\mathbf{y}_{k}$

$$
\mathbf{y}_{k}=\sum_{j=1}^{K} \sqrt{p_{j}} \sqrt{h_{j}} b_{j} \mathbf{a}_{j} s_{j}^{(k)}+\mathbf{n}_{k}
$$

which represents the chip matched filtered samples at the $k$ th chip interval across the $L$ antenna array elements. Over one bit period, $T_{b}=N T_{c}$, we collect a set of $N L$-dimensional

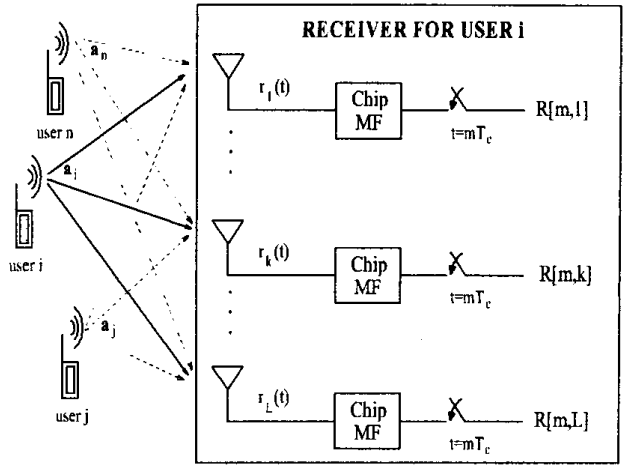

Figure 1. Received signal model for user $i$, $R[m, k]$ denotes the $(m, k)$ th element of the received signal matrix $R$.

vectors $\left\{\mathbf{y}_{k}, 1 \leq k \leq N\right\}$ which we can arrange in an $N \times L$ matrix $\mathbf{R}=\left[\mathbf{y}_{1}, \mathbf{y}_{2}, \cdots, \mathbf{y}_{N}\right]^{\top}$ (Figure 1):

$$
\mathbf{R}=\sum_{j=1}^{K} \sqrt{p_{j}} \sqrt{h_{j}} b_{j} \mathbf{s}_{j} \mathbf{a}_{j}^{\top}+\mathbf{N}
$$

These $L N$ observation samples can also be seen as a collection of $L N$-dimensional vectors, i.e., $\mathbf{R}=\left[\mathbf{z}_{1}, \mathbf{z}_{2}, \cdots, \mathbf{z}_{L}\right]$, where $\mathbf{z}_{l}$ denotes the observation vector consisting $N$ chip matched filtered samples at the output of the $l$ th antenna element and is expressed as

$$
\mathbf{z}_{l}=\sum_{j=1}^{K} \sqrt{p_{j}} \sqrt{h_{j}} b_{j} \mathbf{s}_{j} a_{j}^{(l)}+\mathbf{n}_{l}
$$

In (4), $\mathbf{N}$ is the matrix that represents the spatially and temporally white noise, i.e., $E\left[N_{k l}^{*} N_{m n}\right]=\sigma^{2} \delta_{k m} \delta_{l n}$, where $(.)^{*}$ denotes the conjugate of a complex number. We label user $i$ as the desired user and the other users as interferers.

In contrast with references $[2,17,18]$ where the values of all small-scale fading coefficients are assumed to be perfectly tracked at the receiver, in this paper, we will assume that only large-scale fading coefficients of the interferers can be tracked. Similar to [19] we make the following assumptions:

(I) The receiver has all side information regarding the desired user; its temporal signature, amplitude, and large and small-scale fading coefficients

(II) The following side information is available for each interferer: temporal signature, amplitude, large-scale fading coefficient. The receiver is not able to track the small-scale fading coefficients of the interferers. 
We model the small-scale fading coefficients of all users across all antennas, $a_{j l}, j=1, \cdots, K, l=1, \cdots, L$, as i.i.d. complex 0 -mean Gaussian random variables with variance $1 / L(1 / 2 L$ per complex dimension $)$.

\section{Combined Temporal-Spatial Filters}

The detection of the information bit of the desired user is done by taking the sign of the real part of the decision statistic which is found by combining the entries of the observation matrix $\mathbf{R}$ by using a matrix filter $\mathbf{X}_{i}[18]$. Thus, the decision statistic, $d_{i}$, is the output of a two dimensional linear filter $\mathbf{X}_{i}$ :

$$
d_{i}=\sum_{k=1}^{N} \sum_{l=1}^{L}\left[X_{i}\right]_{k l}^{*} R_{k l}=\operatorname{tr}\left(\mathbf{X}_{i}^{H} \mathbf{R}\right)
$$

where $\operatorname{tr}(\cdot)$ and $(\cdot)^{H}$ are the trace and the hermitian transpose operations, respectively.

We aim for a filter that yields the minimum mean squared error (MMSE) between $d_{i}$ and $b_{i}$, under the assumptions given in Section 2. That is, we want to find a matrix filter $\overline{\mathbf{X}}_{i}$ such that

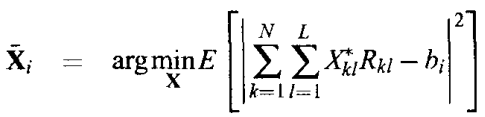

$$
\begin{aligned}
& =\arg \min _{\mathbf{X}} E\left[\left|\operatorname{tr}\left(\mathbf{X}^{H} \mathbf{R}\right)-b_{i}\right|^{2}\right]
\end{aligned}
$$

where $\operatorname{tr}(\cdot)$ and $(\cdot)^{H}$ are the trace and the hermitian transpose operations on a matrix, respectively.

The optimization problem (7) can be converted to an optimization problem with vector variables for easier manipulation [11]. Let $\mathbf{r}$ be the long vector obtained by stacking the columns of the received signal matrix, $\mathbf{R}$.

$$
\mathbf{r}=\sum_{j=1}^{K} \sqrt{\bar{p}_{j}} \sqrt{h_{j}} b_{j} \mathbf{q}_{j}+\overline{\mathbf{n}}
$$

where $\mathbf{q}_{j}$ is the combined temporal-spatial signature of user $j$ and is constructed by stacking columns of $\mathbf{s}_{j} \mathbf{a}_{j}^{\top}$ as a long vector of size $L N$. The MMSE problem then can be reformulated as follows:

$$
\overline{\mathbf{x}}_{i}=\arg \min _{\mathbf{s}} E\left[\left|\mathbf{x}^{H} \mathbf{r}-b_{i}\right|^{2}\right]
$$

Then, the solution to the optimization problem (9) is given as [14]:

$$
\overline{\mathbf{x}}_{i}=\left(E\left[\mathbf{r r}^{H}\right]\right)^{-1} E\left[b_{i} \mathbf{r}\right]
$$

Under assumptions (I) and (II), we have $E\left[b_{i} \mathbf{r}\right]=\sqrt{p_{i} h_{i}} \mathbf{q}_{i}$, and

$$
E\left[\mathbf{r r}^{H}\right]=\sum_{j \neq i} p_{j} h_{j} E\left[\mathbf{q}_{j} \mathbf{q}_{j}^{H}\right]+p_{i} h_{i} \mathbf{q}_{i} \mathbf{q}_{i}^{H}+\sigma^{2} \mathbf{I}
$$

Furthermore, $E\left[\mathbf{q}_{j} \mathbf{q}_{j}^{H}\right]$ becomes a block-diagonal matrix,

$$
E\left[\mathbf{q}_{j} \mathbf{q}_{j}^{H}\right]=\frac{1}{L}\left(\begin{array}{cccc}
\mathbf{s}_{j} \mathbf{s}_{j}^{\top} & \mathbf{0} & \ldots & \mathbf{0} \\
\mathbf{0} & \mathbf{s}_{j} \mathbf{s}_{j}^{\top} & \ldots & \mathbf{0} \\
\vdots & \vdots & \ddots & \vdots \\
\mathbf{0} & \mathbf{0} & \ldots & \mathbf{s}_{j} \mathbf{s}_{j}^{\top}
\end{array}\right)
$$

and using (12), the matrix inversion lemma and some straightforward algebra, it follows that

$$
\overline{\mathbf{x}}_{i}=k\left(\begin{array}{c}
a_{i 1} \mathbf{A}^{-1} \mathbf{s}_{i} \\
a_{i 2} \mathbf{A}^{-1} \mathbf{s}_{i} \\
\vdots \\
a_{i L} \mathbf{A}^{-1} \mathbf{s}_{i}
\end{array}\right)
$$

where

$$
k=\frac{\sqrt{p_{i} h_{i}}}{1+p_{i} h_{i}\left(\sum_{l=1}^{L}\left|a_{i l}\right|^{2}\right) \mathbf{s}_{i}^{H} \mathbf{A}^{-1} \mathbf{s}_{i}}
$$

and

$$
\mathbf{A}=\sum_{j \neq i} \frac{1}{L} p_{j} h_{j} \mathbf{s}_{j} \mathbf{s}_{j}^{\top}+\sigma^{2} \mathbf{I}
$$

Note that the matrix filter $\overline{\mathbf{X}}_{i}$ is constructed by taking every $N$ element of $\overline{\mathbf{x}}_{i}$ and putting as a column to $\overline{\mathbf{X}}_{i}$. The following observation is immediate.

Observation $1 \overline{\mathbf{X}}_{i}$, the optimum joint temporal-spatial MMSE receiver under assumptions (I) and (II) is of the form $\overrightarrow{\mathbf{X}}_{i}=\mathbf{c}_{i} \mathbf{w}_{i}^{\top}$ with

$$
\begin{aligned}
\mathbf{c}_{i} & =k \mathbf{A}^{-1} \mathbf{s}_{i} \\
\mathbf{w}_{i} & =\mathbf{a}_{i}
\end{aligned}
$$

where $k$ and $\mathbf{A}$ are given by (14) and (15), respectively.

Note that in Observation $1, \mathbf{c}_{i}$ is in the form of a temporal MMSE filter for a single antenna receiver [5] with interferers' powers multiplied by the variance of the small-scale fading coefficients, $1 / L$, whereas $\mathbf{w}_{i}$ is the simple spatial matched filter, i.e., the maximum-ratio-combiner (MRC).

In contrast with the above case where interferers' smallscale fading coefficients are not known at the receiver, for the case where small-scale fading coefficients of all users are tracked at the receiver, i.e., all $\mathbf{q}_{j}, j=1, \cdots, K$ are known, the optimum MMSE receiver is given by

$$
\hat{\mathbf{x}}_{i}=k^{\prime}\left(\sum_{j \neq i} p_{j} h_{j} \mathbf{q}_{j} \mathbf{q}_{j}^{H}+\sigma^{2} \mathbf{I}\right)^{-1} \mathbf{q}_{i}
$$

and thus requires an $N L \times N L$ matrix inversion $[11,18]$. This matrix filter can be expressed as

$$
\hat{\mathbf{X}}_{i}=\sum_{k=1}^{m} \mathbf{c}_{i k} \mathbf{w}_{i k}^{\top}
$$


where $1 \leq \boldsymbol{m} \leq \min (N, L)$ is the rank of $\hat{\mathbf{X}}_{i}$. Motivated by the potential high complexity of this optimum receiver, constrained optimum filters, that solve the optimization problem (7) by forcing $\hat{\mathbf{X}}_{i}$ to lie in the rank-1 matrix space $(m=1)$, i.e., $\hat{\mathbf{X}}_{i}=\mathbf{c}_{i} \mathbf{w}_{i}^{\top}$, were proposed in [18]. The corresponding optimum $\mathbf{c}_{i}$ and $\mathbf{w}_{i}$ did not admit closed forms and had to be found by iterative methods [18]. Observation 1 states that when small-scale fading coefficients of the interferers are not tracked at the receiver, the best joint temporal-spatial processor is a separable, i.e., a rank-1, filter which employs a temporal MMSE filter followed by a simple MRC. Therefore, the following simple observation can be readily made.

Observation 2 Under assumptions (I) and (II), the joint optimum and the constrained optimum filters coincide, and have well defined closed forms.

Another interesting observation relates the cascaded temporal-spatial filter, proposed in [17] to the optimum filter under assumptions (I) and (II) as follows. The structure of the cascaded temporal-spatial receiver is to combine the received chip samples at the output of each array element in the MMSE sense independent of each other followed by a spatial filter that combines the resulting vector in the MMSE sense. Although this filter structure cannot in general minimize the end-to-end MSE as is done by the joint optimum MMSE filter, it can outperform the constrained optimum (rank-1) MMSE filter [17]. The derivation of this filter entails designing an MMSE filter for the $l$ th antenna branch observing $\mathbf{z}_{l}$ given in (5) only. If the small-scale fading coefficients of all users are known at the receiver, this results in [17]

$$
\mathbf{c}_{l}=\sqrt{p_{i} h_{i}} a_{i}^{(l)}\left(\sum_{j=1}^{K}\left|a_{j}^{(l)}\right|^{2} p_{j} h_{j} \mathbf{s}_{j} \mathbf{s}_{j}^{\top}+\sigma^{2} \mathbf{I}\right)^{-1} \mathbf{s}_{i}
$$

followed by a spatial MMSE combiner

$$
\mathbf{w}=\sqrt{p_{i} h_{i}}\left(\sum_{j=1}^{K} p_{j} h_{j} \tilde{\mathbf{a}}_{j} \tilde{\mathbf{a}}_{j}^{H}+\Lambda\right)^{-1} \tilde{\mathbf{a}}_{i}
$$

where $\Lambda=\operatorname{diag}\left\{\sigma^{2}\left(\mathbf{c}_{l}^{H} \mathbf{c}_{l}\right)\right\}$ and $\tilde{a}_{j}^{(l)}=\left(\mathbf{c}_{l}^{H} \mathbf{s}_{j}\right) a_{j}^{(l)}$. Note that $c_{\text {l's }}$ differ from each other due to the particular realization of small-scale fading coefficients of the users to the different antennas. The overall matrix filter can be expressed as

$$
\mathbf{X}_{i}^{\mathrm{TS}-\mathrm{CMMSE}}=\sum_{l=1}^{L} w_{l} \mathbf{c}_{l} \mathbf{e}_{l}^{\top}
$$

where $\mathbf{e}_{l}$ is the $L$ vector which has 1 in its $l$ th entry and zeros elsewhere. This matrix filter can be of rank up to $L$.

In the case where small-scale fading coefficients of the interferers are not known at the receiver, the temporal
MMSE filter to process the samples at the output $l$ th antenna, $\mathbf{z}_{l}$, can be shown to be

$$
\mathbf{c}_{l}=k_{l} \mathbf{A}^{-1} \mathbf{s}_{i}
$$

with

$$
k_{l}=\frac{\sqrt{p_{i} h_{i}} a_{i l}}{1+p_{i} h_{i}\left|a_{i l}\right|^{2} \mathbf{s}_{i}^{H} \mathbf{A}^{-1} \mathbf{s}_{i}}
$$

and $\mathbf{A}$ is given in (15). Further algebra reveals that the $l$ th element of the spatial MMSE combiner cascaded with these temporal combiners is given by

$$
w_{l}=k \frac{a_{i l}}{k_{l}}
$$

where $k$ is given by (14). It is easily shown that the overall matrix filter then can be expressed as

$$
\mathbf{X}_{i}^{\text {TS-CMMSE }}=\mathbf{c}_{i} \mathbf{w}_{i}^{\top}
$$

where $c_{i}$ and $\mathbf{w}_{i}$ are the same as those in (16) and (17).

Observation 3 The cascaded temporal-spatial MMSE and the joint optimum MMSE filters are identical under assumptions (I) and (II), i.e., cascaded MSE minimization is equivalent to end-to-end MMSE.

\section{Discussion}

We have shown that, in a CDMA system employing an antenna array at the base station receiver, under the assumptions that the receiver is able to track the temporal signatures and the large scale fading coefficients of all users, and the small-scale fading effects of only the desired user, the joint optimum temporal-spatial MMSE filter, the rank-1 constrained optimum temporal-spatial filter and the temporal-spatial cascaded MMSE filter all coincide and therefore have identical performance.

Naturally, when less information is available at the receiver regarding the interferers, the performance is compromised. An important performance comparison criterion is the bit error rate (BER). Unfortunately, for general system parameters, it is difficult to derive analytical results for the BER, rendering this comparison intractable. Commonly the BER is expressed as a function of the signal-to-interference ratio (SIR). It was reported in [9] that this approximation is particularly accurate when MMSE receivers are employed. Figure 2 shows the SIR of the desired user after temporalspatial processing versus the common signal-to-noise ratio (SNR) of the interferers for an example system with $K=8$ users, $N=16$ and $L=4$. The SNR of the desired user is $10(10 \mathrm{~dB})$. When fading coefficients of the interferers are available, i.e., when we have perfect side information 


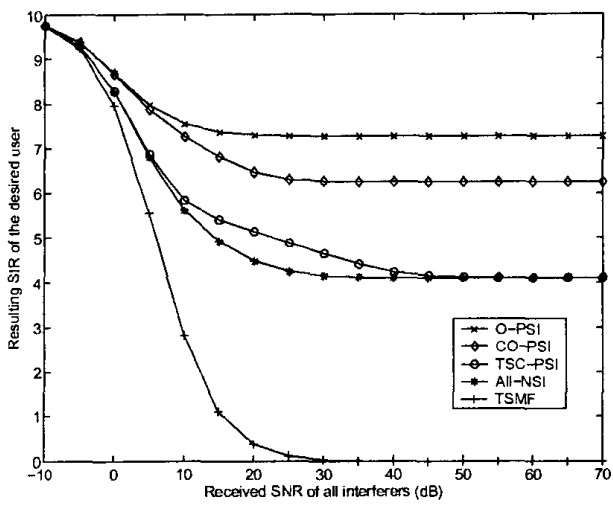

Figure 2. $K=8, L=4, N=16$. O: optimum, CO: constrained optimum, TSC: temporal-spatial cascaded (PSI stands for perfect state information), All-NSI: O, CO and TSC with no side information (NSI), TSMF: matched filter in both temporal and spatial domains.

(PSI), the joint optimum receiver (O-PSI) clearly outperforms the constrained optimum detector (CO-PSI), and the cascaded optimum detector (TSC-PSI). When these values are not available however (no side information (NSI)), all three receivers reduce to the same receiver (All-NSI), optimum and constrained optimum receivers suffering more severely compared to the cascaded optimum receiver. The resulting receiver, in the near-far situation, uses the available signal space dimensions in the temporal domain to decorrelate the interferers via temporal processing and then applies MRC across antennas. The performance of temporal-spatial matched filter is also plotted (TSMF).

We note that the filter structures when fading coefficients are correlated and/or partial side information is available would be different from those presented here. Finally, of current interest is the large system performance of temporalspatial filters under several side information and fading scenarios.

\section{References}

[1] X. Bernstein and A. M. Haimovich. Space-time optimum combining for CDMA communications. Wireless Personal Communications, (3):73-89, 1996.

[2] S. V. Hanly and D. N. C. Tse. Resource pooling and effective bandwidths in CDMA systems with multiuser receivers and spatial diversity. IEEE Transactions on Information Theory. To appear. http://degas.eecs.berkeley.edu/ $\sim$ dtse/pub.html.
[3] R. Kohno, H. Imai, M. Hatori, and S. Pasupathy. Combination of an adaptive array antenna and a canceller of interference for direct-sequence spread-spectrum multiple-access system. IEEE JSAC, 8(4):675-682, May 1990.

[4] R. Lupas and S. Verdú. Linear multiuser detectors for synchronous code-division multiple-access channels. IEEE Trans. Info. The, 35(1):123-136, January 1989.

[5] U. Madhow and M. L. Honig. MMSE interference suppression for direct-sequence spread-spectrum CDMA. IEEE Trans. Comm., 42(12):3178-3188, December 1994.

[6] R. A. Monzingo and T. W. Miller. Introduction to Adaptive Arrays. Wiley, 1980.

[7] A. F. Naguib, A. J. Paulraj, and T. Kailath. Capacity improvement with base-station antenna arrays in cellular CDMA. IEEE Trans. Vehic. Tech., 43(3):691-698, August 1994.

[8] A. J. Paulraj and C. B. Papadias. Space-time processing for wireless communications. IEEE Signal Proc. Mag., 14(6):49-83, November 1997.

[9] H. V. Poor and S. Verdú. Probability of error in MMSE multiuser detection. IEEE Trans. Info. The., 43(3):858-871, May 1997.

[10] F. Rashid-Farrokhi, L. Tassiulas, and K. J. R. Liu. Joint power control and beamforming in wireless networks using antenna arrays. IEEE Transactions on Communications, 46(10):247-256, 1998.

[11] V. G. Subramanian and U. Madhow. Blind demodulation of direct-sequence CDMA signals using an antenna array. In CISS, 1996.

[12] S. Ulukus and R. D. Yates. Adaptive power control and MMSE interference suppression. ACM Wireless Networks, Special Issue on Multiuser Detection in Wireless Communications, 4(6):489-496, November 1998.

[13] S. Ulukus and R. D. Yates. Iterative signature adaptation for capacity maximization of CDMA systems. In 36th Annual Allerton Conference on Communications, Control and Computing, September 1998.

[14] S. Verdú. Multiuser Detection. Cambridge University Press, 1998.

[15] X. Wang and H. V. Poor. Space-time multiuser detection in multipath CDMA channels. IEEE Trans. Signal Proc., 47(9):2356-2374, September 1999

[16] T. Wong, T. M. Lok, J. S. Lehnert, and M. D. Zoltowski. A linear receiver for direct-sequence spread-spectrum multiple-access systems with antenna arrays and blind adaptation. IEEE Trans. Info. The, 44(2):659-676, March 1998.

[17] A. Yener, R. D. Yates, and S. Ulukus. Combined temporal and spatial filter structures for CDMA systems. In IEEE Vehicular Technology Conference, September 2000.

[18] A. Yener, R. D. Yates, and S. Ulukus. Interference management for CDMA systems through power control, multiuse detection, and beamforming. IEEE Trans. Comm., 2001. To appear. http://www.eecs.lehigh.edu/ $\sim$ yener.

[19] J. Zhang, E. Chong, and I. Kontoyiannis. Unified spatial diversity combining and power allocation schemes for CDMA systems. In IEEE Global Communications Conference, pages 1365-1369, December 2000. 